\title{
Skeletal Muscle Metastases and Inferior Vena Cava Involvement in a Patient with Clear Cell Renal Cell Carcinoma and Sarcomatoid Differentiation
}

\author{
Călin Molnar', Octavian-Sabin Tătaru², Lucian Mărginean³ ${ }^{3}$ Angela Borda4 \\ 1 Surgery Clinic No. I, County Emergency Clinical Hospital, University of Medicine and Pharmacy, Tîrgu Mureș, Romania \\ 2 Clinic of Urology, County Emergency Clinical Hospital, University of Medicine and Pharmacy, Tîrgu Mureș, Romania \\ 3 Department of Radiology, County Emergency Clinical Hospital, University of Medicine and Pharmacy, Tîrgu Mureș, Romania \\ 4 Department of Pathology, County Emergency Clinical Hospital, University of Medicine and Pharmacy, Tîrgu Mureș, Romania
}

\section{CORRESPONDENCE \\ Octavian-Sabin Tătaru \\ Str. Gheorghe Marinescu nr. 1 \\ 540103 Tîrgu Mureș, Romania \\ Tel +40758919891 \\ E-mail: sabin.tataru@gmail.com}

\section{ARTICLE HISTORY}

Received: 1 August, 2016

Accepted: 2 September, 2016

Călin Molnar • Str Gheorghe Marinescu nr 50 540136 Tîrgu Mureș, Romania. Tel +40 265212111 Lucian Mărginean - Str. Gheorghe Marinescu nr. 50, 540136 Tîrgu Mureș, Romania. Tel +40 265212111

Angela Borda • Str. Gheorghe Marinescu nr. 38 540139 Tîrgu Mures, Romania. Tel +40 265215551

\begin{abstract}
Introduction: Renal cell carcinoma has a propensity to propagate into the renal vein and inferior vena cava. A small percentage has distant metastasis at presentation. Pulmonary, hepatic, cerebral and bone metastases are common, but skeletal muscle involvement is rare. Case presentation: We present the case of a 51-year-old patient complaining of right flank pain, gross hematuria and a painful left laterothoracic mass. Preoperative examination revealed a tumor in the inferior pole of the right kidney, thrombosis of the right renal vein that extended into the inferior vena cava and a left laterothoracic tumor. We decided on a preoperative digital subtraction angiography and selected embolization of the laterothoracic mass. We performed right radical nephrectomy with vena cava thrombus excision and excision of the left laterothoracic tumor. The pathological examination revealed a clear cell renal carcinoma with sarcomatoid differentiation of the right kidney. Metastases with the above features were noticed in the right adrenal gland and in the skeletal muscle of the chest wall. Conclusions: The surgical resection of large renal tumors with associated thrombus within the inferior vena cava is challenging to any surgeon. The preoperative embolization of the metastatic tumor is helpful in the reduction of pain and intraoperative blood loss.
\end{abstract}

Keywords: inferior vena cava thrombosis, renal vein thrombosis, sarcomatoid clear cell renal cell carcinoma, skeletal muscle metastasis

\section{INTRODUCTION}

Three percent of the solid tumors in adults are found to be renal cell carcinomas (RCC), with the highest incidence at an age between 50 and 70 years. Pulmonary $(50 \%)$, lymphatic nodes $(35 \%)$, hepatic $(30 \%)$, bone $(30 \%)$ and adrenal (5\%) metastases are the most frequent in this type of neoplasia. ${ }^{1}$

Clear cell renal cell carcinoma (CCRCC) metastasizing into muscles is an atypical discovery. Satake et al. concluded that only 32 cases of skeletal muscle 


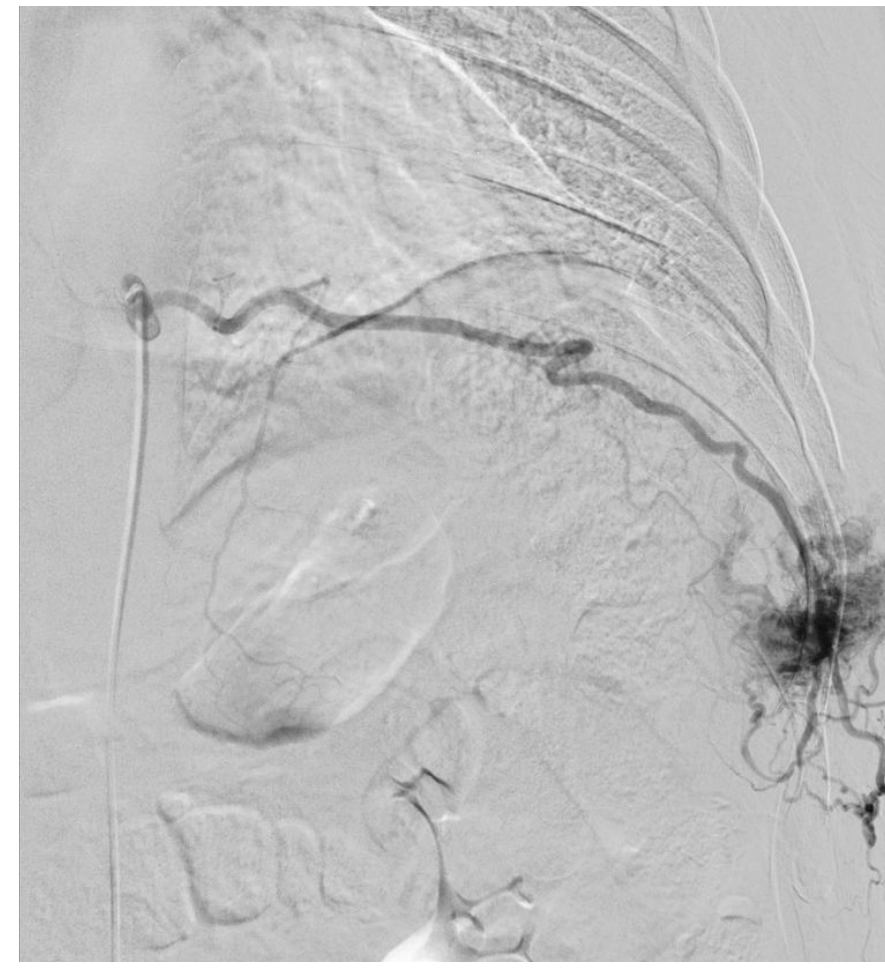

FIGURE 1. Performing a microcatheterization of the intercostal artery and embolization with polyvinyl alcohol, 150-300 $\mu \mathrm{m}$ particles, to total tumor stasis and devascularization.

metastasis from RCC had been reported until 2009, and Sountoulides et al. discovered 3 more. Patients with RCC that present with metastatic involvement of the skeletal muscles are exceptional, thus making the present case report as set apart from the rest. ${ }^{2}$

Sarcomatoid RCC (sRCC) has yet to be completely described. Therefore, it is a very aggressive form of renal neoplasia, due to the incomplete understanding of its physiopathology and possible form of treatments. Being an aggresive form of cancer, in which patients present in an advanced stage of evolution, sRCC is rather uncommon to medical clinicians that deal with metastatic ailments. ${ }^{3}$

\section{CASE PRESENTATION}

We report the case of a 51-year-old male patient complaining of right flank pain, gross hematuria and a painful left laterothoracic mass that had evolved over the last 6 months. On examination, the general status was good, and on local examination a $10 \times 10 \mathrm{~cm}$ palpable tumor and nonpalpable kidneys were observed. Past personal and family medical history was not significant.

A thoracic, abdominal and pelvic computerized tomography (CT) scan was performed, that described a right renal inferior pole tumor, the presence of a right renal vein throm- bus shortly extending into the inferior vena cava without lymph nodes involvement, and a left latero-thoracic tumor, with no other abnormalities identified on CT scan.

Prior to surgery, the patient underwent an embolization procedure for the laterothoracic tumor. We used the Seldinger technique through a right femoral approach. The tumor vasculature formation of the chest wall was identified, with blood supply from the VIII ${ }^{\text {th }}$ intercostal artery, which was embolized to total tumor stasis and devascularization, followed by pain relief at the tumor site, on the same day (Figure 1).

Under general anesthesia, within 24 hours from the embolization of the thoracic tumor, we performed right nephrectomy and adrenalectomy, longitudinal resection of the inferior vena cava, right longitudinal cavoraphy. There was no drop in the patient's blood pressure during the inferior vena cava approach.

An incision was made in the center of the laterothoracic tumor with the complete macroscopical resection of the tumor situated in the intercostal space between the VIII ${ }^{\text {th }}$ and IX $^{\text {th }}$ rib with minimal blood loss.

The pathological examination revealed a CCRC with sarcomatoid differentiation of the right kidney (Fuhrman 4). The great majority of the tumor was composed of conventional CCRC, with a Fuhrman 2 nuclear grade. About $5 \%$ of the tumor showed a sarcomatoid differentiation, with rhabdoid appearance of the cells. Rather extensive necrosis was present in these areas the tumor. Upon immunohistochemical examination, both conventional and sarcomatoid components expressed CD 10, EMA, AE1/ $\mathrm{AE} 3, \mathrm{CK} 8, \mathrm{CK} 18$ and were negative for CK 7, CK 20, AMACR, CD117 (Figure 2).

The renal vein contained a tumor thrombus and multiple emboli that were present in the small veins of the renal sinus. Metastases with conventional CCRC feature were noticed in the right adrenal gland and in the skeletal muscle of the chest wall.

The patient was further referred to an oncologist for chemotherapy.

\section{DISCUSSION}

It has been reported that approximately $0.4 \%$ of RCC metastasize to skeletal muscle. Even though the skeletal muscle presents an abundant blood supply and accounts for a large surface of the body, metastatic involvement is very exceptional at this site. The presence of peptidic factors preventing the metastasis and muscular contractions dislodging anchored tumor cells provide insight into the rarity of skeletal metastasis. ${ }^{5}$ 

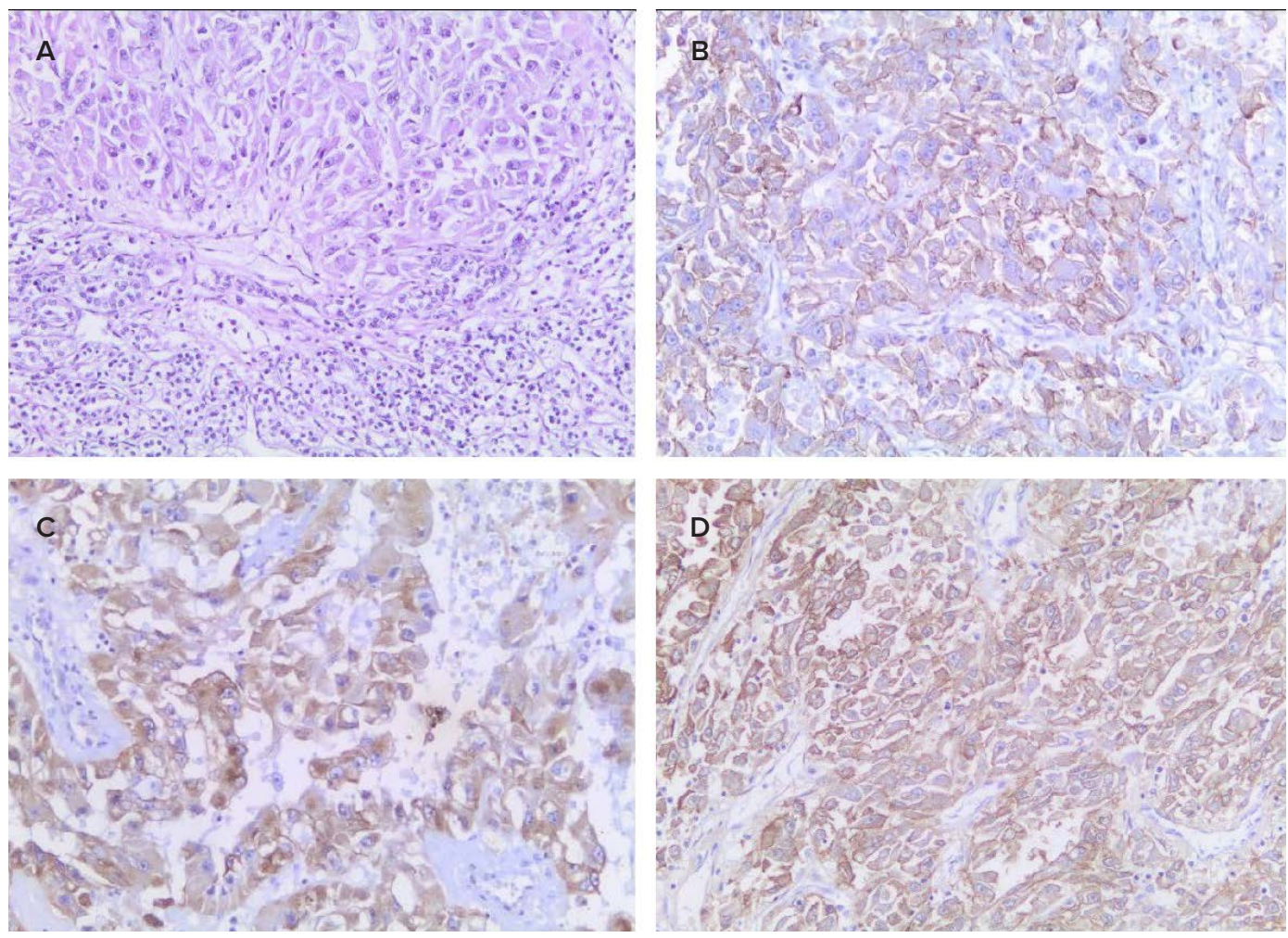

FIGURE 2. A - Sarcomatoid differentiation with rhabdoid appearance merging from Fuhrman 2 CCRC. B - Week positivity of CD 10 in the sarcomatoid component of CCRC. C - Strong positivity of AE1/AE3. D Positivity of EMA with membrane delineation.

The percentage of sarcomatoid differentiation within the tumor seems not to influence overall survival in patients with cM1 disease. ${ }^{8}$ RCC commonly metastasizes to soft tissues as a single soft tissue deposit developing at any point ranging from 6 months to 19 years, the maximum risk being within the initial 5 years after first medical contact. In our reported case, the skeletal muscle metastasis was synchronous with the primary tumor. ${ }^{6}$

Renal cell carcinoma metastases present a highly developed vasculature, with increased bleeding described during the surgery. Major hemorrhage can be prevented during surgery. Transarterial embolization was found to be useful in alleviating pain immediately. The procedure can be safely executed, without including non-target embolization, if a previous angiography is performed for an exact anatomical evaluation of the vessels, as well as a precise cathether insertion in the arteries that supply the tumor. This is why we decided on preoperative embolization of the left laterothoracic tumor. ${ }^{7}$

\section{CONCLUSIONS}

Cases with sarcomatoid renal cell carcinoma present a poor prognosis due to its increased rate of recurrence and high mortality and morbidity, indicating the need for more effective systemic therapies. Further trials are needed to discover the reason why skeletal muscle metastases develop in such a small percentage of patients with RCC and to evaluate the transarterial embolization benefits from an oncological point of view.

\section{COMPETING INTERESTS}

The authors declare that they have no conflict of interests and that they have no financial interests related to the material in the manuscript.

\section{FUNDING}

We state that the authors did not receive any funding for this manuscript.

\section{CONSENT}

Written informed consent was obtained from the patient for the publication of this report and any accompanying images. A copy of the written consent is available for review by the Editor-in-chief of this journal. 


\section{REFERENCES}

1. Togral G, Arıkan M, Gungor S. Rare skeletal muscle metastasis after radica nephrectomy for renal cell carcinoma: evaluation of two cases. J Surg Case Rep. 2014;2014(10):rju101.

2. Sountoulides P, Metaxa L, Cindolo L. Atypical presentations and rare metastatic sites of renal cell carcinoma: a review of case reports. J Med Case Reports. 2011;5:429.

3. Shuch B1, Bratslavsky G, Linehan WM, Srinivasan R. Sarcomatoid renal cell carcinoma: a comprehensive review of the biology and current treatment strategies. Oncologist. 2012;17(1):46-54
4. Vipin L, Lohiya S, Windsor K. A large thigh mass: a blood clot or a rare skeletal muscle metastasis from renal cell carcinoma. SpringerPlus. 2013;2:399

5. Ali SH, Chughtai H, Alali F, Diaczok B, Verardi M. Wrist drop: an atypical presentation of renal cell carcinoma. Am J Med Sci. 2011;342(2):170-173.

6. Owen RJT. Embolization of musculoskeletal bone tumors. Semin Intervent Radiol. 2010; 27(2):111-123.

7. Kim T, Zargar-Shoshtari K, Dhillon J. Using percentage of sarcomatoid differentiation as a prognostic factor in renal cell carcinoma. Clin Genitourin Cancer. 2015;13(3):225-230. 